\title{
Revisiting the etiological aspects of dissociative identity disorder: a biopsychosocial perspective
}

This article was published in the following Dove Press journal:

Psychology Research and Behavior Management

2 May 2017

Number of times this article has been viewed

\author{
Vedat Șar' \\ Martin J Dorahy ${ }^{2}$ \\ Christa Krüger ${ }^{3}$ \\ 'Department of Psychiatry, Koç \\ University School of Medicine, \\ Istanbul, Turkey; ${ }^{2}$ Department of \\ Psychology, University of Canterbury, \\ Christchurch, New Zealand; \\ ${ }^{3}$ Department of Psychiatry, University \\ of Pretoria, Pretoria, South Africa
}

Correspondence: Vedat Şar Department of Psychiatry, Koç University School of Medicine, Rumelifeneri Yolu, 34450 Sariyer, Istanbul, Turkey Email vsar@ku.edu.tr

\begin{abstract}
Dissociative identity disorder (DID) is a chronic post-traumatic disorder where developmentally stressful events in childhood, including abuse, emotional neglect, disturbed attachment, and boundary violations are central and typical etiological factors. Familial, societal, and cultural factors may give rise to the trauma and/or they may influence the expression of DID. Memory and the construction of self-identity are cognitive processes that appear markedly and centrally disrupted in DID and are related to its etiology. Enduring decoupling of psychological modes may create separate senses of self, and metamemory processes may be involved in interidentity amnesia. Neurobiological differences have been demonstrated between dissociative identities within patients with DID and between patients with DID and controls. Given the current evidence, DID as a diagnostic entity cannot be explained as a phenomenon created by iatrogenic influences, suggestibility, malingering, or social role-taking. On the contrary, DID is an empirically robust chronic psychiatric disorder based on neurobiological, cognitive, and interpersonal non-integration as a response to unbearable stress. While current evidence is sufficient to firmly establish this etiological stance, given the wide opportunities for innovative research, the disorder is still understudied. Comparison of well-selected samples of DID patients with non-dissociative subjects who have other psychiatric disorders would further delineate the neurobiological and cognitive features of the disorder, whereas genetic research on DID would further illuminate the interaction of the individual with environmental stress. As such, DID may be seen as an exemplary disease model of the biopsychosocial paradigm in psychiatry.
\end{abstract}

Keywords: dissociation, childhood trauma, neurobiology, family dysfunction, social factors, cultural factors, dissociative identity disorder

\section{Introduction}

Dissociative identity disorder (DID) is multifactorial in its etiology. Whereas psychosocial etiologies of DID include developmental traumatization and sociocognitive sequelae, biological factors include trauma-generated neurobiological responses. Biologically derived traits and epigenetic mechanisms are also likely to be at play. At this point, no direct examination of genetics has occurred in DID. However, it is likely to exist, given the genetic link to dissociation in general and in relation to childhood adversity in particular. ${ }^{1,2}$

Studies conducted in various countries led to a consensus about prevalences of DID: ${ }^{3} 5 \%$ among psychiatric inpatients, $2-3 \%$ among outpatients, and $1 \%$ in the general population. Prevalences appear heightened among adolescent psychiatric outpatients and in the psychiatric emergency unit. ${ }^{4,5}$ The latter points to the acute transient crisis 
situations that may be superimposed on an underlying DID. Such crises usually serve as a diagnostic window for the clinician in conditions when core symptoms of DID remain dormant until a stressful event triggers a more prominent manifestation.

Self-report instruments such as the Dissociative Experiences Scale (DES), ${ }^{6}$ the Dissociation Questionnaire (DISQ), ${ }^{7}$ and the Multidimensional Inventory of Dissociation $(\mathrm{MID})^{8}$ serve as potential screening measures for patients with DID. Clinician-administered instruments such as the Dissociative Disorders Interview Schedule (DDIS) ${ }^{9}$ and the Structured Clinical Interview for DSM-IV Dissociative Disorders (SCID-D) ${ }^{10}$ guide the clinician in making a firm diagnosis of DID.

DID is a stable diagnostic category when the primary components of the disorder are considered: the existence of dissociative identities, confusion about and alterations between them, amnesia, and experiences of depersonalization and derealization. ${ }^{11}$ The foundation of dissociative identities is thought to be divisions or dissociations within the individual at the level of identity or the personality. Dissociation or a breakdown of integration between psychobiological aspects and systems that make up the totality of the person's functioning can occur at the level of sensation (e.g., temporary/functional blindness), cognition (e.g., amnesia), affect (e.g., numbing), behavior (e.g., temporary/functional paralysis), and consciousness (e.g., ego-observing depersonalization), among others. ${ }^{12}$

Yet, when all these systems come together to underpin and maintain a person's identity, and dissociation occurs at this (identity/personality) level, it creates dissociative identities. Here, separate organized systems of functioning, with their own unique perspective on the world and who they are, appear to co-exist within the individual. ${ }^{13}$ Each of these identities has their own first-person perspective or experience of self-consciousness. ${ }^{14-16}$ Consequently, each of these identities reports their own subjective experiences and memories, their own sense of agency and will, and their own perspective on who they are. ${ }^{14}$ They often report being unaware of other identities or report amnesia for experiences that presumably occurred when other identities were engaging in executive actions.

This paper examines the etiological factors of DID, setting them around the current most widely accepted primary driver of the psychopathology: developmental traumatization. Secondarily, family and sociocultural factors and further cognitive and neurobiological disturbances emerge in tandem with this primary factor. These etiological factors have also been the subject of an accumulating body of research in the field of dissociation in general and dissociative disorders other than DID. However, in this paper, only studies directly addressing DID have been considered for examination.

\section{Developmental traumatization}

DID is currently understood as a chronic complex posttraumatic developmental disorder where adverse experiences usually begin in early childhood and in which the dissociative identities result from the child's inability to develop and maintain a unified sense of self across various discrete behavioral states. ${ }^{17-23}$ (See also the literature referred to in the next paragraph.) Identity alterations observed in DID may also be considered as an elaborated version of trauma-related mental intrusions and avoidance that corresponds to the basic mechanism of post-traumatic stress disorder (PTSD). ${ }^{24-26}$ While the dynamics are similar, DID has discrete identities with their own first-person perspective (multiple "I" selves) and breaks in consciousness between these identities, which do not occur in PTSD. ${ }^{4}$

More severe and earlier-onset child abuse appears to differentiate DID from other disorders. ${ }^{27,28}$ Large-scale clinical and epidemiological studies in the United States, Australia, Turkey, Puerto Rico, the Netherlands, Germany, and Canada have consistently found that DID is linked to antecedent chronic abusive experiences in childhood, typically at the hands of an attachment figure. ${ }^{29-31}$ A large representative sample of women from the general population in Turkey $(\mathrm{N}=994)$ was evaluated in three stages: after completing a self-report measure of dissociation, two groups of participants with high and low scores were administered the DDIS by a researcher blind to scores, followed by blinded clinical examination. The researchers were able to identify four cases of DID, all of whom reported childhood abuse and/or neglect. ${ }^{32}$

Dalenberg et $\mathrm{al}^{20}$ calculated Ross and Ness ${ }^{33}$ comparison of physical and sexual abuse in DID patients and controls, finding effect sizes of $0.74-0.78$. By using corroborating documentation from hospital, police, and child protection agencies or witnesses, several studies have confirmed histories of severe abuse in DID. ${ }^{29,34,35}$ In most clinical series, childhood abuse and/or neglect is reported by $90-100 \%$ of the patients directly during the study examination. ${ }^{36,37}$ Dissociative amnesia for childhood events may prevent such reports by some of the patients. ${ }^{38}$

Milder presentations of DID are sometimes associated with traumatization that is covert, such as enduring severely dysfunctional communication and relationship styles in family members, including subtle forms of emotional neglect. 
In a study conducted on a large group of college students, Şar et $\mathrm{al}^{39}$ demonstrated that emotional neglect predicted a dissociative disorder (including DID) diagnosis. Krüger and Fletcher ${ }^{37}$ demonstrated that self-reported emotional neglect by biological parents or siblings in childhood was the strongest individual predictor of an adult diagnosis of a dissociative disorder (including DID) in psychiatric patients (out of all other combinations of abuse type and abuserabused relational ties).

Some data and theory suggest that disorganized attachment style may also underpin the development of DID. ${ }^{40-45}$ Bowlby ${ }^{46}$ proposed that inadequate care-seeking interactions with primary caregivers could lead the infant to develop multiple internal representations of self and attachment figures (which he called internal working models [IWM]). Main and Hesse $^{47}$ identified disorganized attachment developing from a relational context where the child who is seeking safety and comfort is frightened by the caregiver whom the child is pursuing for soothing or the child frightens the caregiver, thereby impeding connection. Contradictory IWM develop to represent the caregiver as dangerous and safe at the same time. These models activate in contexts of abuse to motivate protection from the same caregiver to whom safety seeking is also sought. Early-onset abuse and/or neglect by a relational figure is associated with disorganized attachment (and disorganized attachment may heighten the likelihood of abuse experiences). Hence, disorganized attachment may provide a foundation for DID. ${ }^{40,48}$

DID patients often feel very isolated/lonely, in the sense that they believe they are the only one in the universe who is "different" from others and that they do not understand themselves. Hence, they experience depersonalization and derealization that may go back to their childhood. ${ }^{49}$ DePrince et $\mathrm{al}^{50}$ found that alienation was the only cognitive appraisal variable to differentiate DID from PTSD. While the groups had similar appraisals of shame, betrayal, self-blame, anger, and fear, the DID participants had higher appraisal of themselves as experiencing alienation. This construct is associated with feeling alone, disconnected, and different.

Abuse and neglect may activate feelings of alienation, isolation, and loneliness, and such experiences may compound the impact of trauma and the development and maintenance of DID. If the necessary relational support to enable constructive processing of specific abuses is absent, the child is impeded in their ability to make sense of these experiences through narrative and the containment of the affective states activated, thus inhibiting the integration of the abuse with other autobiographical experiences. Consequently, the representations of abuse/neglect experiences remain isolated from integration, and with further incidences and isolation, the child's ability to develop an ordinary sense of self-in-relation-to-others, based on a coherent narrative that includes the abuse experiences, is impeded and dissociative identities may begin to form. Interpersonal and internal phobias (e.g., phobias of other dissociative identities) then interfere with cooperation toward change, integration, and growth. ${ }^{51,52}$

High hypnotizability - itself a non-pathological, genetically derived capacity - has also been proposed to be a necessary diathesis for DID. ${ }^{53}$ Although patients with DID have higher hypnotizability than those with other mental disorders, higher hypnotizability is also found in patients with chronic refractory post-traumatic states in general. ${ }^{54-56}$ Hence, data are required to determine the degree it represents a diathesis for DID that may reflect part of the biological contribution to the development of the disorder in the presence of other necessary factors.

\section{Family, society, and culture}

DID can be found in all cultural settings. ${ }^{57}$ Cultural processes influence the development and phenomenology of DID. ${ }^{58,59}$ The role of culture may be divided into two components: as the origin of trauma and as modifier of expression of the disorder. Although childhood abuse and neglect require the presence and actions of "perpetrators", they can occur only in a suitable environment. This environment is characterized by denial, boundary violations, reality distortions, paranoia, narcissism, and dramatic posturing, which usually serves the purpose of maintaining the family structure. These features and dynamics may derive from psychological, relational, and economic needs of one or both of the parents, as well as oppressive traditions that do not allow a dissolution of marriage and other contextual issues in the family. ${ }^{60}$

Dysfunctions in the family may partly originate from parents' own traumatic antecedents that lead to inter-generational transmission of developmental stress as reported in the context of the "apparently normal (dissociative) family" by Öztürk and Şar. ${ }^{61}$ In their empirical study, family members of patients with DID and related dissociative disorders reported frequent mood swings, intense anger and inability to control anger, transient dissociative experiences or paranoid ideas, and identity confusion more frequently than controls. Some of these features were correlated with certain types of childhood trauma in this group. For example, frequent mood swings were associated with all types of childhood trauma except sexual abuse, and identity confusion was correlated with emotional abuse. ${ }^{61}$ 
In such a family with subclinical dissociative characteristics, individuals can interchange their social roles over time, alternating between being a victim, abuser, and rescuer. ${ }^{62}$ Depending on their own traumatic past, or on their current interaction between each other and with their children, the parents may maintain trust and present themselves in a positive role ("angelic", affectionate/compassionate parent), but they can turn to an abusive parenting style (angry, aggressive, insistent) at any time. The changing attitudes of their parents and the marital discord will often cause contradictory feelings within the children. Family members often feel trapped, first being unable to leave in the midst of a crisis as it is not safe. Then, they do not leave the family when the crisis is over and the need to escape has vanished, as the atmosphere becomes less threatening and more settled. Third, in an environment of neglect, chaos may be an opportunity for making contact with others in the unit. ${ }^{61}$ Upon direct traumatization early in life, the ever-changing roles in an enduring family system continue to push children and adolescents to utilize a dissociative adaptation style in a period sensitive to the establishment of a stable identity.

Betrayal trauma ${ }^{63}$ (i.e., trauma perpetrated by someone the victim relies on, e.g., by a primary caregiver ${ }^{64}$ is common in such family systems that are characterized by secrets and denial. Betrayal trauma theory suggests that dissociative amnesia is an adaptive response to childhood abuse that allows for survival by enabling the child to maintain attachment to an abusive figure who is also vital to his or her development. A recent study by Kaehler and Freyd ${ }^{65}$ found that higher betrayal traumas are associated with greater "borderline" characteristics that are common in DID as secondary features that do not necessarily point to an underlying personality disorder. ${ }^{24,38,39,49}$

As a possible example of the influence of culture as a modifier of the expression of DID, in a comparison of Turkish and Dutch patients with DID, large differences existed between the two groups in meeting borderline personality disorder criteria. ${ }^{27,36}$ Namely, Turkish patients reported intense anger and lack of control of this emotion, chronic feelings of emptiness and boredom, efforts to avoid abandonment, and intense but unstable relationships more frequently than Dutch patients. In turn, Dutch patients reported frequent mood swings, physically self-damaging acts, identity confusion, and impulsive and unpredictable behavior more frequently than Turkish patients. While such differences seem to originate from cultural factors, some types of affect dysregulation, possibly independent of cultural environment, were common to both groups. In another study - possibly as an indicator of the relational nature of the local culture Turkish adolescent outpatients with dissociative disorders (including DID) differed from non-dissociative psychiatric outpatients on heightened prevalence of concurrent separation anxiety disorder. ${ }^{4}$

Families constitute a subsystem in the broader context of society and culture. As presented in a South African example of culture as modifier of the expression of DID, ${ }^{66}$ dissociation may help individuals or communities to survive in a world of conflicting messages, where conflict is often interpersonal/ cultural/societal in nature, rather than primarily intrapsychic. The resultant fluctuating self-states - that might have been diagnosed as the dissociative identities of DID in a different cultural context - may be tolerated as normal expressions of societal conflict in a culture where conflicting ideologies pervade everyday life and where a normal sense of self is deeply dependent on a certain level of connectedness with others. Covering a subtler version of such systemic influence as well, Şar and Öztürk ${ }^{67}$ proposed a model of sociocognitive adaptation to developmental traumatization at the cost of internal detachment of the individual ("functional dissociation of the self").

The so-called sociocognitive model of DID (e.g., Lynn et $\mathrm{al}^{68}$ ) went beyond recognizing the influence of sociocognitive factors on the development and phenomenology of DID. This model suggested that media reports, a high level of social knowledge about DID, influential and suggestive therapists, as well as patients' own suggestibility, cognitive distortions and fantasy proneness all led patients to believe (wrongly) that they had dissociative identities. This view of DID markedly contrasts with the post-traumatic model of DID (outlined earlier), which proposes that dissociative identities are the primary results of early trauma and the relational, cognitive, emotional, and neurobiological consequences of it (along with other related factors as outlined in this paper) rather than primarily the result of social and cognitive forces. Moreover, the presence of sociocognitive forces does not provide any proof for iatrogenesis. Yet, sociocognitive and trauma models are not entirely contradictory, ${ }^{69}$ as the trauma model, e.g., argues that social and cultural factors influence the presentation, but not typically the creation, of dissociative identities..$^{20,69-71}$ In fact, societal conditions themselves may also be the source of traumatic antecedents as observed in oppressive communities and traditions. $^{72}$ 


\section{Cognitive etiological models and factors}

Memory and the construction of self-identity are cognitive processes that appear markedly and centrally disrupted in DID, such that the discriminating symptoms of the disorder are the experience of amnesia for autobiographical events and the presence (or experience) of non-integrated, dissociative identities. Consequently, cognitive processes are implicated in the etiology of DID. The way cognition is organized and the appraisals and beliefs associated with what autobiographical information is available for retrieval may give rise to dissociative identities and amnesia between them. The development of a sense of self is predicated on the collection of life experiences that are encoded as occurring to the self. ${ }^{73}$ Thus, the construction of self is underpinned by episodic and semantic autobiographical memories laid down with autonoetic consciousness (i.e., this experience happened to me, and when I remember, it has the felt sense that I experienced it). ${ }^{74}$

As such, an apparent etiological feature of dissociative identities is associated with the breakdown between encoding experienced events (i.e., the functioning of the human memory system) and perceiving ownership of those events as part of autobiographical experience (i.e., self-referencing the events). That is, there is a breakdown between memory and sense of self. Dissociative identities have their origin in memories of experienced events being owned or feeling like they relate to self in some identities but not others. Thus, different identities draw on, and feel ownership of, 1) different memories, 2) different aspects of the same experience, or 3) overlapping memories/experiences with different appraisals and narratives. Dissociative identities and the different bundles of owned memorized experience that characterize them may differ markedly from each other on lower order characteristics, such as physiological arousal (e.g., heart and respiration rate, blood pressure), affective tone, and neurobiological correlates (e.g., dominant brain activation), and higher order characteristics, such as the ability to have ownership of lived experiences, as well as appraise and narrate them. ${ }^{14,18,75}$

\section{Dissociative identities}

Kennedy et al have elucidated a cognitive perspective on the etiology of DID, drawing on the work of Beck. ${ }^{76-78}$ Beck $^{76}$ proposed that the personality is made up of "modes", which contain cognitive, affective, behavioral, and physiological representations or schema for encoding experience and responding to internal and environmental demands. For example, a woman may have a "mother" mode that contains how she thinks, feels, behaves, and gets physiologically activated when caring for her child. These modes and the schema that make them up are mobilized by cues detected by what Beck called "orienting schema", which appraise the internal and external landscape.

Cues identified by the orienting schema to indicate the requirements of child care will initiate, for instance, the mother mode. Under normal circumstances, this mode will be connected with other modes, such as the mode processing and responding to demands about the woman's job or modes associated with defensive operations such as verbal aggression. All these modes are umbrellaed under the "conscious control system" that provides a means of unifying the multiple modes into a more integrated sense of self(i.e., a sense of "I" who has multiple ways of being and negotiating the world, via different modes or different "Me's"). DID arises when modes become decoupled and exist in smaller, more isolated pockets (e.g., modes associated with relationship functioning, being a mother, and being a partner become decoupled from modes associated with occupational functioning). ${ }^{77-79}$

This decoupling creates multiple conscious control systems that capture the different and discontinuous arrays of modes, each having their own way of representing that aspect of self, based on the modes that make them up. The etiology of DID then, from a cognitive perspective, is related to the more enduring decoupling or dissociation of the association between modes and the development of different or unique first-person perspectives, or separate senses of self, based on the make-up of these non-coupled clusters of modes.

\section{Amnesia}

Attempts to account for amnesia reported across different dissociative identities in clinical practice ${ }^{63,76}$ initially drew on state-dependent memory phenomena as more controlled research began investigating memory anomalies across different identities. State-dependent memory refers to the superior recall of information encoded and retrieved in the same emotional state. ${ }^{80}$ Alternatively, it is the process by which an individual fails to remember mundane information (e.g., where they left their phone) because they encoded the experience (putting their phone on the mantelpiece) in a different state of mind to that in which they are trying to retrieve the information. They may have encoded the information in a highly aroused state following a conflict with their partner and now in a calm state are attempting to find their phone.

Perhaps, the most elaborated framework of this theory for dissociative disorders is encompassed in Putnam's ${ }^{75}$ discrete behavioral states model. He argues that initially 
through biological decrees (need to feed, sleep) and then through experience (e.g., exposure to traumatic stress), different behavioral states come online and/or develop to support survival and promote adaptation to the environment. ${ }^{18}$ These behavioral states differ not only in their manifest behavior (e.g., sleeping, feeding, socializing, escaping danger via fleeing) but also in all other psychophysiological dimensions, including arousal level, heart rate, motivation, affective tone, thought patterns and content, appraisals, and brain area activation.

Putnam $^{75}$ argues that trauma exposure leads to the development of discrete behavioral states that differ vastly in their psychophysiological make-up from other states of consciousness, giving rise to pathological dissociative symptoms and, in the most extreme case, dissociative identities. In the case of amnesia, it is proposed to be evident in situations where differences in state-dependent characteristics between encoding and retrieval are most distinct, so, e.g., retrieval failure when a state characterized by relatively minimal arousal is asked about an experience encoded in a high state of arousal (e.g., an episode of incestuous abuse).

In DID, amnesia across identities is accounted for by encoding in one identity that has a very different psychophysiological make-up than the identity attempting to retrieve the information. As a result, amnesia would be predicted to be more likely across identities that are vastly different in their biopsychosocial characteristics. This theory draws on mechanisms evident in normal cognitive functioning (i.e., state-dependent memory) to explain amnesia across dissociative identities. It is proposed to account for findings in research that suggested that information encoded in one dissociative identity may not be available in a dissociative identity reporting amnesia for that information. ${ }^{81}$

Recent research examining amnesia across dissociative identities has required an elaboration of earlier theories in an attempt to address the etiology of amnesia in dissociative identities. This work has found, at least in patients presenting for treatment, that memories residing in another dissociative identity for which amnesia is perceived are actually available for retrieval or at least on objective assessment appear accessible. ${ }^{82}$ This has prompted the question of whether amnesia across identities in DID is a cognitive process (i.e., associated with encoding characteristics and/or retrieval anomalies) or a metacognitive process (e.g., no encoding or retrieval deficits but a belief that content is not available). This latter explanation attempts to account for findings that representations of experience in memory appear to be accessible for retrieval in dissociative identities that believe they have no memory of such material.

Metacognitive processes, or more specifically metamemory processes, are involved in the monitoring, control, and appraisal of memories. They monitor for memories that fit goal-directed aims and assess them for subjective accuracy (e.g., the confidence in which the details retrieved feel accurate), they control which information is best to volunteer, and they subjectively appraise the ability to remember. ${ }^{83}$

Huntjens et al draw on metacognitive processes to account for recent empirical findings, suggesting that amnesia in DID is not the result of being unable to access information but the result of believing that such information is inaccessible. She and her colleagues argued that, "[m]etamemory problems may lead patients not to acknowledge correctly retrieved material, which is believed to 'belong' to other identities, or indeed choose an incorrect answer alternative following the belief that the correct answer alternative cannot be known if the material that one is tested for was learned [or experienced] by another identity". ${ }^{84}$ The etiology of interidentity amnesia from this perspective is a metamemory belief that the material is unavailable rather than it being unavailable. ${ }^{85}$ It resides in metamemory processes rather than in deficit memory functions. Consequently, the etiology of dissociative amnesia across identities may lie in information being encoded in a state (i.e., identity) that the retrieving identity, 1) believes they have no access to, and therefore gives up the search for such information, despite its availability, 2) appraises retrieval success as minimal, or 3) monitors with no confidence that retrieved information belongs to the self and therefore discards it.

While it remains to be examined empirically, the reported experience of amnesia across identities in DID may have its origin in retrieval states (i.e., dissociative identities) being psychobiologically distinct from encoding states (i.e., different dissociative identities). Such state-dependent processes may disrupt the search for and appraisal of encoded information (i.e., metamemory processes).

\section{Neurobiology of DID}

Studies on the neurobiology of DID are in their infancy. They have focused mainly on two issues: differences between dissociative identities within patients with $\mathrm{DID}^{86}$ and differences between patients with DID and controls (the controls being either healthy subjects or other psychiatric patients). There are both structural and functional neuroimaging findings that demonstrate differences between dissociative patients and non-clinical populations. However, the specificity of these 
findings for DID would hinge on future comparisons with patients with other psychiatric disorders and whether the structural anomalies, e.g., are a cause or consequence of DID.

In positron emission tomography (PET) studies, when compared to a dissociative identity focused on the tasks of daily living, a dissociative identity focused on trauma memories and defensive operations showed increased cerebral blood flow in the amygdala, insular cortex, somatosensory areas in the parietal cortex, and the basal ganglia, as well as in the occipital and frontal regions and anterior cingulate. ${ }^{87,88}$ In a subsequent PET study, healthy controls simulating distinct dissociative identities were unable to reproduce the same network patterns as the DID patients. ${ }^{86}$

In the quantitative electro-encephalogram (QEEG) study by Lapointe et al, ${ }^{89}$ variability between identity states involved mostly beta activity in the frontal and temporal lobes. Hopper et $\mathrm{al}^{90}$ demonstrated that the average alpha coherence on QEEG was lower for "alter" identities than for "host" identities (i.e., the identity predominantly engaging with the external world) in five DID patients in some temporal, frontal, parietal, and central regions.

In an functional magnetic resonance imaging (fMRI) study, women with DID and matched healthy female actors were compared in their responses (assessed both in "host" and "emotionally laden" dissociative identities) when consecutively exposed to masked neutral and angry faces. ${ }^{91}$ Differences between "host" and "emotionally laden" identities in DID patients and between DID and simulating controls were generally larger for neutral than angry faces. In DID, compared to "host", "emotionally laden" dissociative identities were associated with more activation of the parahippocampal gyrus. Following neutral faces and compared to the "emotionally laden" identity in controls, "emotionally laden" identity in DID had more activation in brainstem, face-sensitive regions, and motor-related areas. There were neither significant withingroup differences nor significant between-group differences in state anxiety. Controls were not able to simulate genuine "host" and "emotionally laden" dissociative identities.

A structural MRI study established that DID patients have smaller hippocampi and amygdalae than normal controls. ${ }^{92}$ Ehling et $\mathrm{al}^{93}$ also found reduced volumes in the parahippocampal gyrus of individuals with DID and strong correlations between reduction of parahippocampal volume and both cognitive-emotional and sensorimotor dissociation, in comparison with normal controls. In two single photon emission computerized tomography (SPECT) studies, DID patients exhibited orbitofrontal hypoperfusion in comparison with normal controls conducted in "host" identities. ${ }^{94,95}$ Bilaterally increased perfusion in prefrontal regions and occipital areas accompanied this in one of these studies. ${ }^{95}$ In the other one, the "host" identity showed increased perfusion in the left (dominant hemisphere) lateral temporal region compared to healthy controls. ${ }^{94}$ This lateralization was not replicated in a follow-up study. ${ }^{95}$

Notwithstanding the possible effect of psychiatric comorbidity as a confounding factor (hence, it cannot be considered as specific to DID), the findings concerning orbitofrontal hypoperfusion do not seem to be at odds with the theoretical understanding of developmental neurobiology. Longitudinal neuroimaging studies suggest that the orbitofrontal cortex is one of the last regions in the brain to fully develop in humans. ${ }^{95}$ A tensor-based morphometry investigation indicates that orbitofrontal cortex volumes are smaller in children who have suffered early aberrant parental care in the form of physical abuse and that these volumetric alterations are associated with difficulties children experience in various aspects of their social lives. ${ }^{96}$ The orbitofrontal cortex is a key component of a circuit that aids in adaptation to changing environmental contingencies and plays an important role in the control of emotion and motivational states. In this regard, Schore ${ }^{97}$ reported that there is a relationship between the development of the orbitofrontal cortex, emotion regulation, and attachment. In accordance with these observations and based on a neurodevelopmental approach, Forrest ${ }^{98}$ proposed an "orbitofrontal model" for DID, which integrates and elaborates on theory and research from four domains: the neurobiology of the orbitofrontal cortex and its protective inhibitory role in the temporal organization of behavior, the development of emotion regulation, the development of the self, and experiencedependent maturation of the orbitofrontal cortex. This model hypothesizes that the orbitofrontal cortex plays a critical role in the development of distinct mental states (i.e., dissociative identities) due to its inhibitory functions.

\section{Conclusion and directions for future research}

Understanding the etiology of DID requires integration of trauma exposure, coping, cognitive, neurobiological, systemic, and developmental factors. These include traumatic experiences, family dynamics, child development, and attachment. ${ }^{17,63,99}$ DID develops when a child is exposed to chaos, coercion, and overt severe physical and/or sexual abuse or, alternatively, to "apparently normal" dissociative families often with subtle neglect, disorganized attachment to caregivers, and misattuned communication styles. ${ }^{59}$ While the role of the child's biological capacity to dissociate to an extreme level is unclear yet, there is evidence demonstrating the neurobiological impact of developmental stress. The 
latter converges around an impairment of connectivity in the central nervous system in affected individuals. From a psychological point of view, multiple self-states that do not become integrated over time allow the child to compartmentalize trauma-related cognitions and overwhelming and conflicting feelings of betrayal, terror, love, and shame. ${ }^{13,17}$ Overwhelmed by intense conflicting needs and emotions, the child is unable to integrate discrete behavioral and emotional states into a coherent or relatively integrated self according to the appropriate sociocultural construction of self. ${ }^{17,100}$ Further research on the etiology of DID should be able to link clinical and empirical dimensions while considering the four domains addressed in this paper: i.e., developmental traumatization, family and sociocultural factors, cognitive functioning, and neurobiological anomalies.

\section{Disclosure}

The authors report no conflicts of interest in this work.

\section{References}

1. Wolf EJ, Rasmusson AM, Mitchell KS, Logue MW, Baldwin CT, Miller MW. A genome-wide association study of clinical symptoms of dissociation in a trauma-exposed sample. Depress Anxiety. 2014;31(4):352-360.

2. Lochner C, Seedat S, Hemmings SMJ, et al. Dissociative experiences in obsessive-compulsive disorder and trichotillomania: clinical and genetic findings. Compr Psychiatry. 2004;45(5):384-391.

3. Şar V. Epidemiology of dissociative disorders: an overview. Epidemiol Res Int. 2011;2011:8. Article ID 404538.

4. Şar V, Önder C, Kılınçaslan A, Zoroğlu SS, Alyanak B. Dissociative identity disorder among adolescents: prevalence in a university psychiatric outpatient unit. J Trauma Dissociation. 2014;15(4):402-419.

5. Şar V, Koyuncu A, Öztürk E, et al. Dissociative disorders in psychiatric emergency ward. Gen Hosp Psychiatry. 2007;29(1):45-50.

6. Carlson E, Putnam FW. An update on the Dissociative Experiences Scale. Dissociation. 1993;6(1):16-27.

7. Vanderlinden J, Van Dyck R, Vandereycken W, Vertommen H, Jan Verkes R. The dissociation questionnaire (DIS-Q): development and characteristics of a new self-report questionnaire. Clin Psychol Psychother. 1993;1(1):21-27.

8. Dell PF. The Multidimensional Inventory of Dissociation (MID): a comprehensive measure of pathological dissociation. J Trauma Dissociation. 2006;7(2):77-106.

9. Ross CA, Heber S, Norton GR, Anderson G, Anderson D, Barchet P. The dissociative disorders interview schedule: a structured interview. Dissociation. 1989;2(3):169-189.

10. Steinberg M. Structured Clinical Interview for DSM-IV Dissociative Disorders - Revised (SCID-D-R). Washington, DC: American Psychiatric Press; 1994.

11. Steinberg M. Handbookfor the Assessment of Dissociation. A Clinical Guide. Washington, DC: American Psychiatric Publications; 1995.

12. Braun BG. The BASK (behavior, affect, sensation, knowledge) model of dissociation. Dissociation. 1998;1(1):4-23.

13. Van der Hart O, Nijenhuis ERS, Steele K. The Haunted Self: Structural Dissociation and the Treatment of Chronic Traumatization. New York, NY: Norton; 2006.

14. Nijenhuis ERS. The Trinity of Trauma: Ignorance, Fragility and Control: The Evolving Concept of Trauma/The Concept and Facts of Dissociation in Trauma. Gottingen: Vandenhoeck \& Ruprecht; 2015.
15. O'Neil JA. Dissociative multiplicity and psychoanalysis. In: Dell PF, O'Neil JA, editors. Dissociation and the Dissociative Disorders: DSM-V and Beyond. New York, NY: Routledge; 2009:287-325.

16. Nijenhuis ERS, Van der Hart O. Dissociation in trauma: a new definition and comparison with previous formulations. J Trauma Dissociation. 2011;12(4):416-445.

17. Putnam FW. Dissociative disorders. In: Cicchetti D, Cohen DJ, editors. Developmental Psychopathology. Vol. 2. New York: Wiley; 2006:657-695.

18. Putnam FW. The Way We Are. How States of Mind Influence Our Identities, Personality and Potential for Change. Los Gatos, CA: International Psychoanalytic Books; 2016.

19. Howell EF, Blizard RA. Chronic relational trauma disorder: a new diagnostic scheme for borderline personality and the spectrum of dissociative disorders. In: Dell PF, O’Neil JA, editors. Dissociation and the Dissociative Disorders: DSM-V and Beyond. New York, NY: Routledge; 2009:495-510.

20. Dalenberg CJ, Brand BL, Gleaves DH, et al. Evaluation of the evidence for the trauma and fantasy models of dissociation. Psychol Bull. 2012;138(3):550-588.

21. Alexander PC, Schaeffer CM. A typology of incestuous families based on cluster analysis. J Family Psychol. 1994;8(4):458-470.

22. Allen JG, Fultz J, Huntoon J, Brethour JR Jr. Pathological dissociative taxon membership, absorption, and reported childhood trauma in women with trauma-related disorders. J Trauma Dissociation. 2002;3(1):89-110.

23. Carlson EB, Dalenberg C, Armstrong J, Daniels JW, Loewenstein R, Roth D. Multivariate prediction of posttraumatic symptoms in psychiatric inpatients. J Trauma Stress. 2001;14(3):549-567.

24. Şar V, Alioğlu F, Akyüz G, Tayakısı E, Öğülmüş EF, Sönmez D. Awareness of identity alteration and diagnostic preference between borderline personality disorder and dissociative disorders. J Trauma Dissociation. Epub 2016 Dec 5:1-17.

25. Şar V. Identity revised: a clinician's perspective on how an identitybased model of mind would look like. In: Sinnott J, editor. Identity Flexibility During Adulthood: Perspectives in Adult Development. New York, NY: Springer. In press 2017.

26. Şar V. Parallel-distinct structures of internal world and external reality: disavowing and re-claiming the self-identity in the aftermath of trauma-generated dissociation. Front Psychol. 2017;8:216.

27. Boon S, Draijer N. Multiple personality disorder in The Netherlands: a clinical investigation of 71 patients. Am J Psychiatry. 1993;150(3):489-494.

28. Dorahy MJ, Middleton W, Seager L, Williams M, Chambers R. Child abuse and neglect in complex dissociative disorder, abuse-related chronic PTSD and mixed psychiatric samples. JTrauma Dissociation. 2016;17(2):223-236.

29. Martinez-Taboas A. Multiple personality in Puerto Rico: analysis of fifteen cases. Dissociation. 1991;4(4):189-192.

30. Middleton W, Butler J. Dissociative identity disorder: an Australian series. Aust N Z J Psychiatry. 1998;32(6):786-804.

31. Ross CA, Norton GR, Wozney K. Multiple personality disorder: an analysis of 236 cases. Can J Psychiatry. 1989;34(5):413-418.

32. Akyüz G, Doğan O, Şar V, Yargıç Lİ, Tutkun H. Frequency of dissociative identity disorder in the general population in Turkey. Compr Psychiatry. 1999;40(2):151-159.

33. Ross CA, Ness L. Symptom patterns in dissociative identity disorder patients and the general population. J Trauma Dissociation. 2010; 11(4):458-468.

34. Coons PM. Confirmation of childhood abuse in child and adolescent cases of multiple personality disorder and dissociative disorder not otherwise specified. J Nerv Ment Dis. 1994;182(8):461-464.

35. Lewis DO, Yeager CA, Swica Y, Pincus JH, Lewis M. Objective 4 documentation of child abuse and dissociation in 12 murderers with dissociative identity disorder. Am J Psychiatry. 1997;154(12): 1703-1710. 
36. Şar V, Yargic LI, Tutkun H. Structured interview data on 35 cases of dissociative identity disorder in Turkey. Am J Psychiatry. 1996;153(10):1329-1333.

37. Krüger C, Fletcher L. Predicting a dissociative disorder from type of childhood maltreatment and abuser-abused relational tie. J Trauma Dissociation. Epub 2017 Feb 23.

38. Şar V, Alioğlu F, Akyüz G, Karabulut S. Dissociative amnesia in dissociative disorders and borderline personality disorder: selfrating assessment in a college population. J Trauma Dissociation. 2014;15(4):477-493.

39. Şar V, Akyüz G, Kuğu N, Ozturk E, Ertem-Vehid H. Axis-I dissociative disorder comorbidity of borderline personality disorder and childhood trauma reports. J Clin Psychiatry. 2006;67(10):1583-1590.

40. Barach PM. Multiple personality disorder as an attachment disorder. Dissociation. 1991;4(3):117-123.

41. Blizard RA. Disorganized attachment, development of dissociated self states, and a relational approach to treatment. J Trauma Dissociation. 2003;4(3):27-50.

42. Byun S, Brumariu LE, Lyons-Ruth K. Disorganized attachment in young adulthood as a partial mediator of relations between severity of childhood abuse and dissociation. JTrauma Dissociation. 2016;17(4): 460-479.

43. Liotti G. Trauma, dissociation, and disorganized attachment: three strands of a single braid. Psychotherapy. 2004;41(4):472-486.

44. Liotti G. A model of dissociation based on attachment theory and research. J Trauma Dissociation. 2006;7(4):55-73.

45. Lyons-Ruth K, Dutra L, Schuder MR, Bianchi I. From infant attachment disorganization to adult dissociation: relational adaptations or traumatic experiences? Psychiatr Clin North Am. 2006;29(1):63-86.

46. Bowlby J. Attachment and Loss. New York, NY: Basic Books; 1969/1982.

47. Main M, Hesse E. Parents' unresolved traumatic experiences are related to infant disorganized attachment status: is frightened or frightening parental behavior the linking mechanism? In: Greenberg M, Cicchetti C, Cummings EM, editors. Attachment in the Preschool Years. Chicago, IL: University of Chicago Press; 1990:161-182.

48. Sachs A. Through the lens of attachment relationship: stable DID, active DID and other trauma-based mental disorders. J Trauma Dissociation. Epub 2017 Feb 23:1-21.

49. Şar V, Alioğlu F, Akyüz G. Depersonalization and derealization in self-report and clinical interview: the spectrum of borderline personality disorder, dissociative disorders, and healthy controls. J Trauma Dissociation. Epub 2016 Sep 28:1-17.

50. DePrince AP, Huntjens RJC, Dorahy MJ. Alienation appraisals distinguish adults diagnosed with DID from PTSD. Psychol Trauma. 2015;7(6):578-582.

51. Frankel AS, O'Hearn TC. Similarities in responses to extreme and unremitting stress: cultures of communities under siege. Psychotherapy. 1996;33(3):485-502.

52. Steele K, van der Hart O, Nijenhuis ERS. Dependency in the treatment of complex posttraumatic stress disorder and dissociative disorders. $J$ Trauma Dissociation. 2001;2(4):79-116.

53. Dell PF. Is high hypnotizability a necessary diathesis for pathological dissociation? J Trauma Dissociation. 2017;18(1):58-87.

54. Frischholz E, Lipman L, Braun B, Sachs R. Psychopathology, hypnotizability, and dissociation. Am J Psychiatry. 1992;149(11): 1521-1525.

55. Spiegel D, Hunt T, Dondershine HE. Dissociation and hypnotizability in posttraumatic stress disorder. Am J Psychiatry. 1988;145(3):301-305.

56. Stutman RK, Bliss EL. Post-traumatic stress disorder, hypnotizability and imagery. Am J Psychiatry. 1985;142(6):741-742.

57. Şar V. The scope of dissociative disorders: an international perspective. Psychiatr Clin North Am. 2006;29(1):227-244.

58. Dorahy MJ. Culture, cognition and dissociative identity disorder. In: Schumaker JF, Ward T, editors. Culture, Cognition and Psychopathology. Westport, CT: Praeger; 2001:157-169.
59. Krüger C. Variations in identity alteration - a qualitative study of experiences of psychiatric patients with dissociative identity disorder. In: Van der Merwe AP, Sinason V, editors. Shattered but Unbroken: Voices of Triumph and Testimony. London: Karnac Books; 2016:133-161.

60. Gold SN. Not Trauma Alone: Therapy for Child Abuse Survivors in Family and Social Context. Philadelphia,PA: Brunner\&Routledge; 2000.

61. Öztürk E, Şar V. "Apparently normal” family: a contemporary agent of transgenerational trauma and dissociation. J Trauma Pract. 2005;4(3-4):287-303.

62. Ross CA. Dissociative Identity Disorder: Diagnosis, Clinical Features, and Treatment of Multiple Personality. 2nd ed. New York, NY: Wiley; 1997.

63. Freyd JJ. Betrayal trauma: traumatic amnesia as an adaptive response to childhood abuse. Ethics Behav. 1994;4(4):307-329.

64. Goldsmith RE, Freyd JJ, DePrince AP. Betrayal trauma: associations with psychological and physical symptoms in young adults. $J$ Interpers Violence. 2012;27(3):547-567.

65. Kaehler LA, Freyd JJ. Borderline personality disorder: a betrayal trauma approach. Psychol Trauma. 2009;1(4):261-268.

66. Krüger C, Sokudela BF, Motlana LM, Mataboge CK, Dikobe AM. Dissociation: a preliminary contextual model. South African J Psychiatry. 2007;13(1):13-21.

67. Şar V, Öztürk E. Functional dissociation of the self: a sociocognitive approach to dissociation. J Trauma Dissociation. 2007;8(4):69-89.

68. Lynn SJ, Lilienfeld SO, Merckelbach H, Giesbrecht T, Van der Kloet D. Dissociation and dissociative disorders: challenging conventional wisdom. Curr Direc Psychol Sci. 2002;21:48-53.

69. Şar V, Krüger C, Martinez-Taboas A, Middleton W, Dorahy MJ. Sociocognitive and posttraumatic models are not opposed. J Nerv Ment Dis. 2013;201(5):439-440.

70. Dalenberg CJ, Brand BL, Loewenstein RJ, et al. Reality vs. fantasy: reply to Lynn et al. (2014). Psychol Bull. 2014;140(3):911-920.

71. Dorahy MJ, Brand BL, Şar V, et al. Dissociative identity disorder: an empirical overview. Aust N Z J Psychiatry. 2014;48(5):402-417.

72. Şar V, Öztürk E. Stimulus deprivation and overstimulation as dissociogenic agents in postmodern oppressive societies. J Trauma Dissociation. 2013;14(2):198-212.

73. Conway MA. Memory and the self. J Mem Lang. 2005;53:594-628.

74. Prebble SC, Addis DR, Tippett LJ. Autobiographical memory and sense of self. Psychol Bull. 2013;139(4):815-840.

75. Putnam FW. Dissociation in Children and Adolescents: A Developmental Perspective. New York, NY: Guilford Press; 1997.

76. Beck AT. Beyond belief: A theory of modes, personality, and psychopathology. In: Salkovskis PM, editor. Frontiers of Cognitive Therapy. New York, NY: Guilford Press; 1996:1-25.

77. Kennedy F. Dissociation, personality and psychopathology: a cognitive approach. In: Kennedy F, Kennerley H, Pearson D, editors. Cognitive Behavioural Approaches to the Understanding and Treatment of Dissociation. Hove, East Sussex: Routledge; 2013:17-39.

78. Kennedy F, Clarke S, Stopa L, et al. Towards a cognitive model and measure of dissociation. J Behav Ther Exp Psychiatry. 2004;35(1):25-48.

79. Kennedy F, Kennerley H. The development of our understanding of dissociation. In: Kennedy F, Kennerley H, Pearson D, editors. Cognitive Behavioural Approaches to the Understanding and Treatment of Dissociation. Hove, East Sussex: Routledge; 2013:1-16.

80. Lang AJ, Craske MG, Brown M, Ghaneian A. Fear-related state dependent memory. Cognition Emotion. 2001;15(5):695-703.

81. Dorahy MJ. Dissociative identity disorder and memory dysfunction: the current state of experimental research, and its future directions. Clin Psychol Rev. 2001;21(5):771-795.

82. Huntjens RJC, Verschuere R, McNally RJ. Inter-Identity autobiographical amnesia in patients with dissociative identity disorder. PLoS One. 2012;7(7):e40580.

83. Koriat A, Goldsmith M. Monitoring and control processes in the strategic regulation of memory accuracy. Psychol Rev. 1996;103(3):490-516. 
84. Huntjens RJC, Peters ML, Woertman L, Bovenschen LM, Martin RC, Postma A. Inter-identity amnesia in dissociative identity disorder: a simulated memory impairment? Psychol Med. 2006;36(6):857-863.

85. Huntjens RJC, Peters ML, Woertman L, Van der Hart O, Postma A. Memory transfer for emotionally valenced words between identities in dissociative identity disorder. Behav Res Ther. 2007;45(4):775-789.

86. Reinders AATS, Willemsen ATM, Vos HPJ, Den Boer JA, Nijenhuis ERS. Fact or factitious? A psychobiological study of authentic and simulated dissociative identity states. PLoS One. 2012;7(6):e39279.

87. Reinders AATS, Nijenhuis ERS, Paans AMJ, Korf J, Willemsen ATM, Den Boer JA. One brain, two selves. Neuroimage. 2003;20(4):2119-2125.

88. Reinders AATS, Nijenhuis ERS, Quak J, et al. Psychobiological characteristics of dissociative identity disorder: a symptom provocation study. Biol Psychiatry. 2006;60(7):730-740.

89. Lapointe AR, Crayton JW, DeVito R, Fichtner CG, Konopka LM. Similar or disparate brain patterns? The intra-personal EEG variability of three women with multiple personality disorder. Clin EEG Neurosci. 2006;37(3):235-242.

90. Hopper A, Ciorciari J, Johnson G, Spensley J, Sergejew A, Stough C. EEG coherence and dissociative identity disorder. J Trauma Dissociation. 2002;3(1):75-88.

91. Schlumpf YR, Nijenhuis ERS, Chalavi S, et al. Dissociative part dependent biopsychosocial reactions to backward masked angry and neutral faces: an fMRI study of dissociative identity disorder. Neuroimage Clin. 2013;3:54-64.
92. Vermetten E, Schmahl C, Lindner S, Loewenstein R, Bremner J. Hippocampal and amygdalar volumes in dissociative identity disorder. Am J Psychiatry. 2006;163(4):630-636.

93. Ehling T, Nijenhuis ERS, Krikke AP. Volume of discrete brain structures in complex dissociative disorders: preliminary findings. Prog Brain Res. 2007;167:307-310.

94. Şar V, Ünal SN, Kızıltan E, Kundakçı T, Öztürk E. HMPAO SPECT study of cerebral perfusion in dissociative identity disorder. J Trauma Dissociation. 2001;2(2):5-25.

95. Şar V, Ünal SN, Ozturk E. Frontal and occipital perfusion changes in dissociative identity disorder. Psychiatry Res. 2007;156(3):217-223.

96. Hanson JL, Chung MK, Avants BB, et al. Early stress is associated with alterations in the orbitofrontal cortex: a tensor-based morphometry investigation of brain structure and behavioral risk. J Neurosci. 2010;30(22):7466-7472.

97. Schore AN. The experience-dependent maturation of a regulatory system in the orbital prefrontal cortex and the origin of developmental psychopathology. Dev Psychopathol. 1996;8:54-87.

98. Forrest KA. Toward an etiology of dissociative identity disorder: a neurodevelopmental approach. Conscious Cogn. 2001;10(3):259-293.

99. Kluft RP. Multiple personality disorder. In: Spiegel D, editor. Dissociative Disorders: A Clinical Review. Lutherville, MD: Sidran Press; 1993:17-44.

100. Schimmenti A, Caretti V. Linking the overwhelming with the unbearable: developmental trauma, dissociation, and the disconnected self. Psychoanal Psychol. 2016;33(1):106-128.
Psychology Research and Behavior Management

\section{Publish your work in this journal}

Psychology Research and Behavior Management is an international, peerreviewed, open access journal focusing on the science of psychology and its application in behavior management to develop improved outcomes in the clinical, educational, sports and business arenas. Specific topics covered in the journal include: Neuroscience, memory and decision making; Behavior
Dovepress

modification and management; Clinical applications; Business and sports performance management; Social and developmental studies; Animal studies. The manuscript management system is completely online and includes a very quick and fair peer-review system, which is all easy to use. Visit http://www. dovepress.com/testimonials.php to read real quotes from published authors. 